This is the accepted manuscript made available via CHORUS, the article has been published as:

\title{
Bright Laser-Driven Neutron Source Based on the Relativistic Transparency of Solids
}

M. Roth, D. Jung, K. Falk, N. Guler, O. Deppert, M. Devlin, A. Favalli, J. Fernandez, D. Gautier, M. Geissel, R. Haight, C. E. Hamilton, B. M. Hegelich, R. P. Johnson, F. Merrill, G. Schaumann, K. Schoenberg, M. Schollmeier, T. Shimada, T. Taddeucci, J. L. Tybo, F. Wagner, S. A. Wender, C. H. Wilde, and G. A. Wurden Phys. Rev. Lett. 110, 044802 - Published 24 January 2013 DOI: 10.1103/PhysRevLett.110.044802 


\title{
A bright, laser-neutron source based on relativistic transparency of solids
}

\author{
M. Roth ${ }^{1,2}$, D. Jung ${ }^{2}$, K. Falk ${ }^{2}$, N. Guler ${ }^{2}$, O. Deppert ${ }^{1}$, M. Devlin ${ }^{2}$, A. Favalli ${ }^{2}$, J. Fernandez ${ }^{2}$, D. Gautier ${ }^{2}$, M. Geissel $^{3}$, R. \\ Haight $^{2}$, C. E. Hamilton ${ }^{2}$, B. M. Hegelich ${ }^{2}$, R. P. Johnson ${ }^{2}$, F. Merrill ${ }^{2}$, G. Schaumann ${ }^{1}$, K. Schoenberg ${ }^{2}$, M.

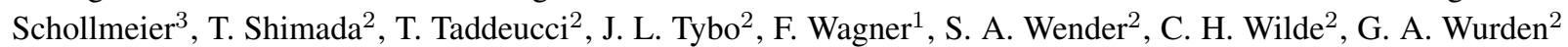 \\ ${ }^{1}$ Institut für Kernphysik,Technische Universität Darmstadt, Schloßgartenstrasse 9, D-64289 Darmstadt, Germany \\ ${ }^{2}$ Los Alamos National Laboratory, Los Alamos, NM 87545, USA and \\ ${ }^{3}$ Sandia National Laboratories, Albuquerque, NM 87185, USA
}

\begin{abstract}
Neutrons are unique particles to probe samples in many fields of research ranging from biology to material sciences to engineering and security applications. Access to bright, pulsed sources is currently limited to large accelerator facilities and there has been a growing need for compact sources over the recent years [1]. Short pulse laser driven neutron sources could be a compact and relatively cheap way to produce neutrons with energies in excess of $10 \mathrm{MeV}$. For more than a decade experiments have tried to obtain neutron numbers sufficient for applications [2-4]. Our recent experiments demonstrated an ion acceleration mechanism based on the concept of relativistic transparency. Using this new mechanism, we produced an intense beam of high energy (up to 170 $\mathrm{MeV}$ ) deuterons directed into a Be converter to produce a forward peaked neutron flux with a record yield, on the order of $10^{10} \mathrm{n} / \mathrm{sr}$. We present results comparing the two acceleration mechanisms and the first short pulse laser generated neutron radiograph.
\end{abstract}

PACS numbers: 52.50.Jm, 52.59.-f, 29.25.Dz, 29.25.-t, 41.75.Jv

Neutrons offer a uniquely different interaction to probe or alter material compared to $\mathrm{x}$-rays or charged particles. Possible applications range from active interrogation of sensitive material [5], nuclear waste transmutation and material testing in fission and fusion reactor research [6]. Moreover, the fundamental disparate dependences of the stopping power with target atomic number allows for complementary information when combined with hard x-rays and energetic charged particles for probing a wide range of target materials. Consequently, the production of penetrating neutrons with energies in excess of $10 \mathrm{MeV}$ is of great interest. Intense pulses of neutrons can be derived from high-energy particle accelerators and high flux beams from fission reactors, but for more portable demands or academic research the size and costs of these devices are preventing their widespread use. It has been established to use high-energy short pulse (HESP) lasers, which are more compact than accelerator systems, to drive intense beams of ions, mainly protons, for years now [7-13]. Consequently attempts have been made to use such systems to produce neutron beams. The most recent experiments [4] used one of the most favorable reactions, where deuterons, accelerated to $\mathrm{MeV}$ energies, are dumped in low- $\mathrm{Z}$ converter targets. The typical scheme to accelerate ions by short pulse lasers is the so-called Target Normal Sheath Acceleration mechanism (TNSA) [7-9]. One major drawback of this scheme is that it always favors the acceleration of surface particles with the highest charge to mass ratio and based on the presence of hydrogenous surface contaminants (water-vapor, hydrocarbons) all the present experiments suffer from a low conversion efficiency for the acceleration of deuterons. Here, we present the first highly efficient neutron production using HESP lasers with ultra high contrast and a recently discovered acceleration mechanism to produce sufficient numbers for fast neutron radiography. With less than a quarter of the laser energy used in previous experiments we obtained not only more than an or- der of magnitude higher neutron yields, but also much higher neutron energies and in a favorable geometry for future applications, i.e. in a forward directed neutron beam.

\section{Relativistic transparency}

The ultra-high contrast of the TRIDENT laser enabled acceleration of deuterons through the Break Out Afterburner (BOA) mechanism, which has been described in detail in $[14,15]$. This mechanism starts as the laser pulse accelerates copious amounts of electrons into and through the opaque target similar to the classical TNSA regime. But then, due to the high intensity and the limited amount of target electrons in the focal volume the target becomes relativistically transparent during the increase of the laser intensity. This is caused by the effective increasing relativistic mass of the oscillating electrons and the reduction of the electron density as their number is limited in thin foils. At this point, close to the peak intensity of the laser pulse, the laser interacts with the entire target volume, continuously resupplying energy to electrons to further accelerate the ions to tens of MeV/amu [16]. Besides generating higher energy particles the main difference of this mechanism is that the entire target volume material is accelerated, independent of the charge to mass ratio. Thus, efficient deuteron acceleration (with a yield one order of magnitude higher than the proton yield) was demonstrated. Beryllium was used as the converter providing a high cross section for neutron production, but minimizing the generation of unwanted high-energy bremsstrahlungs photons. In our experiment neutrons are produced via the nuclear reactions ${ }^{9} \mathrm{Be}(d, n),{ }^{9} \mathrm{Be}(p, n)$, and by the deuteron breakup reaction[17]. For the highest neutron energies of above 150 $\mathrm{MeV}$ also a pre-compound reaction inside the Be nucleus is the most likely origin. While the first reactions are expected 


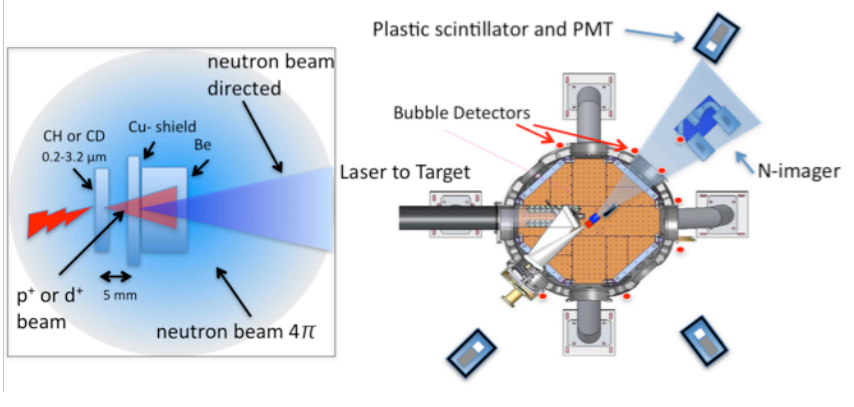

FIG. 1: Experimental setup. (Left): the target consists of $\mathrm{CH} 2$ or $\mathrm{CD} 2$ foils and a $\mathrm{Be}$ converter. The proton or deuteron beam is converted into a spherical $(4 \pi)$ and a directed neutron component. (Right): BTI bubble detectors were placed on the outside of the TRIDENT target chamber to cover different neutron emission angles. Three nToF detectors were used to measure the energy spectrum.

to result in a more or less isotropic neutron emission, the deuteron breakup should result in a forward peaked neutron flux at higher particle energies.

\section{Setup}

The experimental setup is depicted in Figure 1. The experiments were carried out at the Los Alamos National Laboratory 200 TW TRIDENT laser facility, where the temporal contrast (ratio of unwanted laser irradiation compared to the peak intensity) [18] was in excess of $10^{-10}$. In two campaigns we used $f / 3$ and $f / 1.5$ parabolic mirrors close to normal incidence to focus typically 80J of $1.053 \mu \mathrm{m}$ vertically polarized laser light in a clean 600 fs pulse to reach maximum intensities in between $10^{20}$ and $10^{21} \mathrm{~W} / \mathrm{cm}^{2}$. The laser pulse duration and beam parameters were carefully recorded during both campaigns. Plastic $(\mathrm{CH} 2)$ or deuterized plastic (CD2) targets from $200 \mathrm{~nm}$ to $3.2 \mu \mathrm{m}$ thickness were used to generate proton or deuteron beams. Copper activation techniques (nuclear activation imaging spectroscopy, NAIS) [19] were used to measure the proton and deuteron beam parameters for given laser energy and target thickness combinations. A sealed Be converter was placed $5 \mathrm{~mm}$ behind the laser target and protected from the plasma blow-off by a sandwich of three $50 \mu \mathrm{m}$ layers of copper and two layers of $50 \mu \mathrm{m}$ plastic. We used a complete set of diagnostics to fully characterize the neutron source. For the absolute yield measurements we used an array of up to eight standard bubble detectors (BTI [20]) $87 \mathrm{~cm}$ from the target, as these are entirely insensitive to $\mathrm{x}$-rays and electrons. The detectors were re-calibrated to their known non-linear response function to neutron energies in excess of $20 \mathrm{MeV}[21,22]$. The neutron spectral distribution in different directions was measured using several LANSCE neutron Time-of-Flight (nToF) detectors $(10 \mathrm{~cm}$ diameter, $1.88 \mathrm{~cm}$ thick NE102 plastic scintillators coupled to fast $12.5 \mathrm{~cm}$ Hamamatsu R1250A Photo Multiplier Tubes). The $\mathrm{nToF}$ detectors were shielded against the x-rays by up to 25

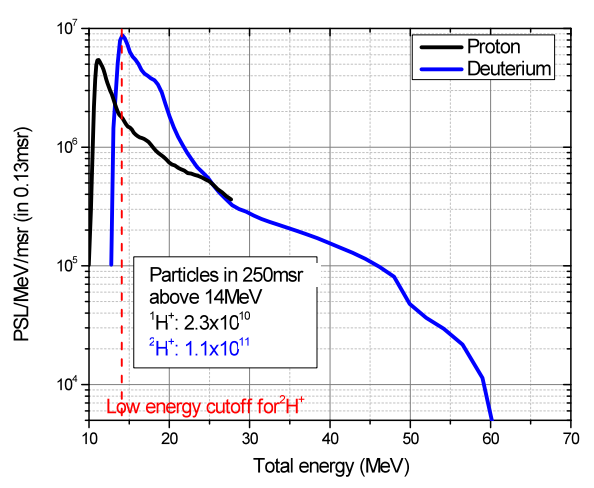

FIG. 2: Ion spectra from a $300 \mathrm{~nm}, 90 \%$ deuterized plastic target. The bulk ion distribution is clearly represented in the spectrum showing the acceleration of the foil volume rather than surface acceleration.

$\mathrm{cm}$ of lead. The distance to the neutron production target was changed between $2.2 \mathrm{~m}$ and $5.7 \mathrm{~m}$ during the experiments and the $\mathrm{nToF}$ detectors covered the $-5,90$ and 180 degree angles with respect to the laser beam. Each nToF detector signal was recorded by a fast digital oscilloscope. The strong, prompt signal from the laser driven $\mathrm{x}$-rays thereby served as a time reference for the neutron energy analysis. The neutron imaging system was placed $2 \mathrm{~m}$ from the target in forward direction. Blocks of tungsten, lead, plastic and steel were used to serve as objects to be radiographed and placed in front of the imager. The iWASP, the activation detectors and a tungsten knife-edge were placed inside the target chamber. A wide angle ion spectrometer (iWASP) [23] complemented the diagnostic setup.

\section{Experiments}

We first tested the BOA mechanism using $\mathrm{CH} 2$ and $\mathrm{CD} 2$ targets and the iWASP [23] spectrometer to measure the yield of deuterons and protons for the two target types (see Figure 2). While for $300 \mathrm{~nm}$ targets the ion distribution resembles the bulk concentration, the $3 \mu \mathrm{m}$ target only showed surface protons with very little deuterium contribution.

Using $\mathrm{CH}$ targets in combination with copper and beryllium converters, we observed an isotropic neutron yield of up to $5 \times 10^{8} \mathrm{n} / \mathrm{sr}$. The beryllium neutron converter target was encapsulated and shielded from the primary target by three layers of copper interleaved with two layers of plastic to absorb the mechanical shock from the expanding primary target. A 5 $\mathrm{cm}$ large block was initially placed in a $5 \mathrm{~cm}$ distance and later replaced by a $6 \times 12 \mathrm{~mm}$ long beryllium rod at $5 \mathrm{~mm}$ distance from the ion source in order to shrink the source size of the neutrons. We then changed from a $\mathrm{CH} 2$ target of $400 \mathrm{~nm}$ to a CD2 target of $3.2 \mu \mathrm{m}$ thickness to test the neutron yield in 


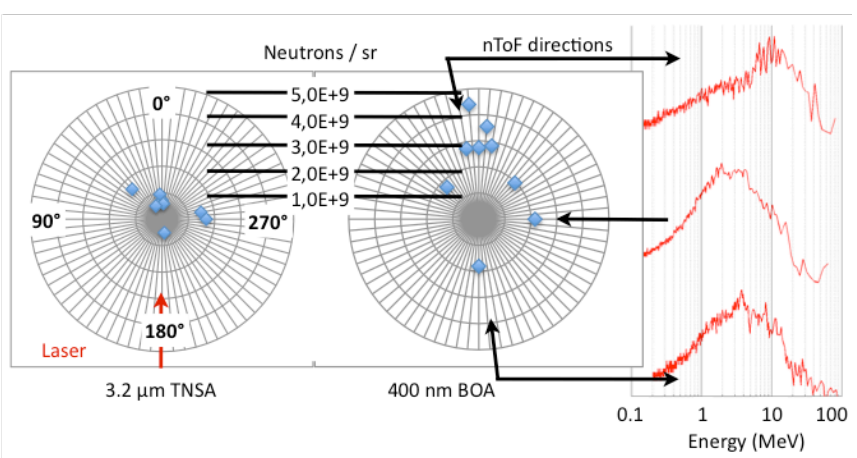

FIG. 3: Neutron Data: Polar plot of an experiment with $3.2 \mu \mathrm{m}$ target (left) compared a $400 \mathrm{~nm}$ target (center). In addition to the isotropic neutron yield in the latter case a strong forward directed yield is visible. The neutron spectrum in forward direction (upper right plot) also shows higher neutron energies.

the classic TNSA regime, which is the dominant acceleration mechanism for a target thickness of several micrometers. In agreement with prior experiments we observed a rather spherical neutron emission. We then changed to $400 \mathrm{~nm} \mathrm{CD} 2$ targets without changing the laser parameters and observed a higher neutron yield of up to $5 \times 10^{9} \mathrm{n} / \mathrm{sr}$ with very different neutron beam signatures (see Figure 3).

The measured neutron yield clearly showed the difference between proton and TNSA dominated experiments and the BOA generated deuteron beams. While the first experiments usually resulted in a spherical emission of neutrons into $4 \pi$, the experiments with ultra-thin targets enabling the BOA regime had an additional strong neutron peak in forward direction. This feature also matched a significant change in the neutron energy spectrum. Note the bump in the upper spectrum in Figure 3 caused by the additional $4 \pi$ component, visible in the lower spectra, which peaks at a few $\mathrm{MeV}$ and extends up to about $15 \mathrm{MeV}$, consistent with the results in [4]. Our second experiment using $10^{21} \mathrm{~W} / \mathrm{cm}^{2}$ resulted in $150 \mathrm{MeV}$ neutrons peaking at $70 \mathrm{MeV}$.

It should also be noted that, when $\mathrm{CH}$ targets have been used, in these experiments the evidence of the BOA regime could be strengthened by observing the in [14] predicted unique spatial beam profile of protons in the high energetic part of the spectrum compared to the much more homogeneous spatial profile produced by the TNSA scheme. Using simple autoradiography techniques the activation of the copper shielding in front of the beryllium converter showed two distinct intensity lobes orthogonal to the laser light polarization (Figure 4). The $\mathrm{Cu}(\mathrm{p}, \mathrm{n}) \mathrm{Zn}$ reaction and the subsequent decay back to $\mathrm{Cu}$ with a half life of $38 \mathrm{~min}$ was used to determine the ion beam imprint by placing the $\mathrm{Cu}$ sample on top of an Image Plate (IP) detector for $1 \mathrm{~min}$ and then digitizing using an IP scanner.

The autoradiography images also provided a test of the source size of the neutrons based on the proton or deuteron beam divergence and its propagation length to the beryllium converter. The maximum neutron flux was observed in the direction of the ion beam with a neutron output of $1 \times 10^{10} n / s r$ in a single

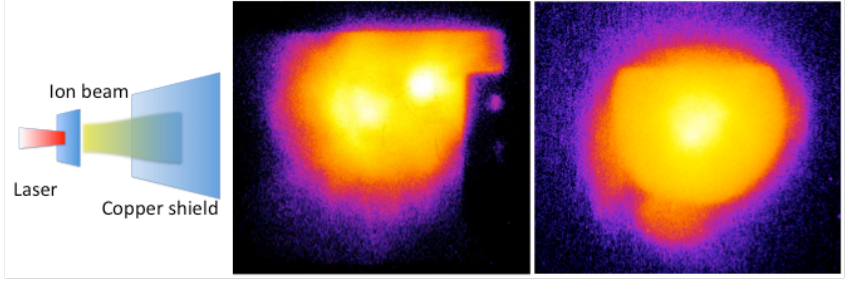

FIG. 4: Ion beam: Autoradiography of the copper shield in front of the beryllium converter at $5 \mathrm{~cm}$ distance from the target (left) using $\mathrm{CH}$ targets. The interesting feature is the distinct two-lobe formation of the most energetic and intense part of the proton beam (center) for a $300 \mathrm{~nm}$ target, characteristic feature of the BOA acceleration scheme. This is different from TNSA (3.2 $\mu \mathrm{m} \mathrm{CH}$ target), where usually homogeneous, circular beams are observed (right). The edge features are caused by mounting structures in the ion beam path.

shot experiment. The flux in all other directions was less than half this maximum value reducing the shielding requirements for future applications. It should be emphasized that in a typical laser generated ion source the initial pulse length of the ion bunch is in the order of several picoseconds [7]. Taking the dispersion of the broad energy spread of the source beam into account the generated neutron beam will have a temporal spread of several hundred of picoseconds.

\section{Radiography with neutrons}

With such an intense, directed and ultra-short neutron beam available we were able to demonstrate the first HESP laserdriven neutron image of a structured object. The main detector for neutron radiography was a fast scintillating fiber array gated neutron imager, developed by LANL for fusion experiments at the National Ignition Facility (NIF) [24]. Neutrons, impacting into a $5 \mathrm{~cm}$ thick fiber array generate light that is transported through the fibers, down collimated by a coherent fiber taper, amplified by a gated micro channel plate and finally detected in a high resolution cooled CCD camera. The scintillator is also sensitive to the large number of $\mathrm{x}$-rays being produced during the initial laser target interaction. With a decay constant (1/e) of $2.5 \mathrm{~ns}$ the x-ray contribution can be used for radiography or excluded from the measurement choosing a specific timing. Moreover, gating the detector well past the decay of the scintillating light caused by the prompt x-rays and limiting the gate width allows for easy selection of the neutron energies, which is of special interest as this allows for the radiography of material imaging different neutron energies. By varying the delay between the laser pulse and the exposure window of the imager, we were able to distinguish between the contribution of instantaneous hard x-ray emission from the primary target and the exposure due to neutrons from several discrete energy intervals. Reducing the distance between the converter and the primary source, the spatial resolution of the image improved. Using a $10 \mathrm{~cm}$ long tungsten edge radiograph, magnified by a factor of two, we deduced a 


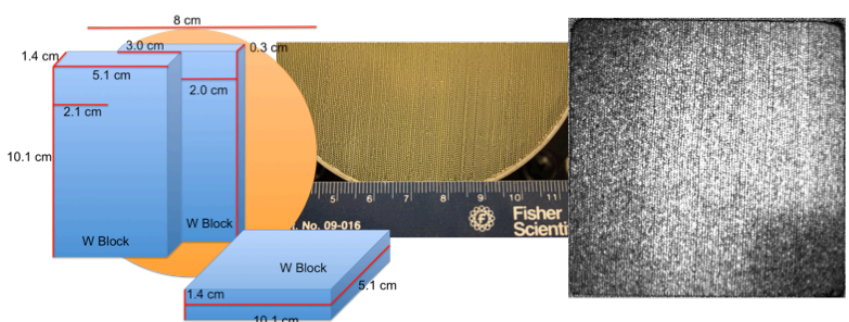

FIG. 5: Neutron image of three tungsten blocks. The left side shows a sketch of the arrangement in front of the scintillating fiber array of the neutron imager. A photograph of the fiber array consisting of a $500 \mu \mathrm{m}$ diameter fiber configuration is shown in the center. A laser pulse with an energy of $75 \mathrm{~J}$ within $800 \mathrm{fs}$ generated a deuteron beam from a $410 \mathrm{~nm} \mathrm{CD} 2$ target. The right side of the figure shows the superposition of two different shots as in figure 6 .
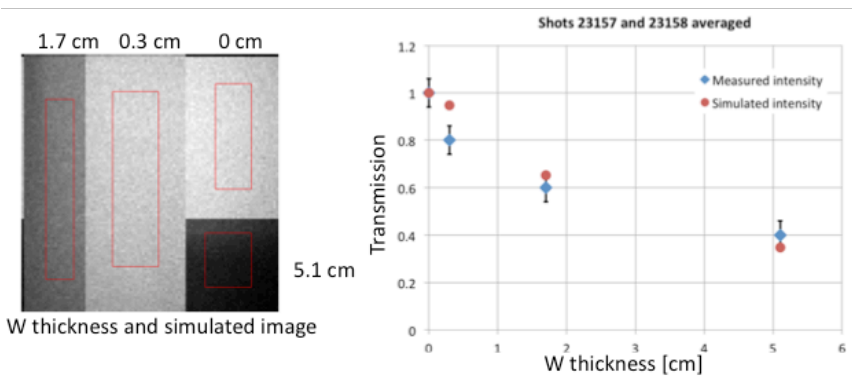

FIG. 6: Comparison between the measured transmissions for different tungsten objects to MCNPX calculations using the measured neutron energy distribution detected by the nTOF detector. The data were averaged over two shots to improve the signal to noise ratio.

projected neutron source size of $3 \mathrm{~mm}$, which is in excellent agreement with the measurements of the ion beam, the converter dimensions, and ion beam straggling in the converter.

A set of tungsten, steel, and plastic samples were then placed into the neutron beam path and their attenuation recorded with the imager. Figure 5 shows an example of one configuration and the resulting neutron image. The scintillating fiber imager started to record $31 \mathrm{~ns}$ after arrival of the x-rays with a gate time of $80 \mathrm{~ns}$. This corresponds to neutron energies between $2.5 \mathrm{MeV}$ and $15 \mathrm{MeV}$. Even with such a large neutron source size of approx. $3 \mathrm{~mm}$ the edge contrast of all three $\mathrm{W}$ blocks is resolved. The attenuation was compared to MCNPX (Version 2.7.0) simulations with enjoy libraries [25] using the measured neutron energy distribution detected by the nToF detectors, the neutron absorption and scattering for the given experimental layout and detector efficiency.

The comparison of the relative and normalized neutron intensity (see Figure 6) shows excellent agreement in the measured and simulated neutron transmission. The deviation of the second data point from the simulation indicates the presence of a hard x-ray background, which is subject to further study. Even in the static case of the probe material, this new method impressively shows its potential for future applications.
In conclusion, these first results prove the concept of using short pulse lasers to drive a compact (few mm size), fast (few $100 \mathrm{ps})$, high energy (>150 MeV) neutron source. Important aspects and advantages for future applications are reduced shielding requirements due to the small source size, the neutron flux can be very high, and a very good temporal resolution. This technique has the potential to open up the use of neutrons in small-scale laboratories and universities and thereby can expand the entire field of neutron research.

\section{Acknowledgments:}

The authors sincerely thank S. Batha and M. Hockaday for their enthusiastic support and gratefully acknowledge the support of the staff at the TRIDENT laser facility at Los Alamos National Laboratory. M. R. is supported by the LANL Rosen Scholar award, O.D. is supported by HIC4FAIR and F.W. is supported by BMBF. This work was performed under the auspices of the US Department of Energy by the Los Alamos National Laboratory under the contract DE-AC5206NA25396. Sandia National Laboratories is a multi-program laboratory managed and operated by Sandia Corporation, a wholly owned subsidiary of Lockheed Martin Corporation, for the U.S. Department of Energy's National Nuclear Security Administration under contract DE-AC04-94AL85000.

[1] Development opportunities for small and medium scale accelerator driven neutron sources IAEA-TECDOC-1439 (2005)

[2] K. Lancaster, et al., Phys Plasmas 7, 3404 (2004)

[3] D.P. Higginson et al., Phys. Plasmas 17, 100701 (2010)

[4] D.P. Higginson et al., Phys. Plasmas 18, 100703 (2011)

[5] A. Buffler, Rad. Phys. Chem. 71, 853 (2004)

[6] L.J. Perkins et al., Nuclear Fusion 40, 1 (2000)

[7] S. P. Hatchett et al., Phys. Plasmas 7, 2076 (2000).

[8] R.A. Snavely et al., Phys. Rev. Lett. 85, 2945 (2000).

[9] S. C. Wilks et al., Phys. Plasmas 8, 542 (2001).

[10] M. Borghesi, et al., Phys. Rev. Lett. 92, 055003 (2004)

[11] J. Fuchs et al., Nature Phys. 2, 48 (2006)

[12] M. Hegelich et al., Nature 439, 441 (2006)

[13] L. Robson et al., Nature Phys. 3, 58 (2007)

[14] L. Yin, et al., Laser and Part. Beams. 24, 291 (2006)

[15] L. Yin L. et al., Phys. Plasmas 14, 056706 (2007)

[16] S. Iizuka et al., Phys. Rev. Lett. 43, 1404 (1979)

[17] http://www.jcprg.org/exfor/

[18] S. Batha et al., Rev. Sci Instrum. 79, $10 \mathrm{~F} 305$ (2008)

[19] M. Günther et al., Fus. Science and Techn. 61, 231 (2012)

[20] Bubble Technology Industries Inc. Calibration of BTI BD-PND neutron detectors. Information note (1996)

[21] R. Olsher et al., Radiat. Protection Dosimetry, 126, 326 (2007)

[22] Smecka F. and Hajek M., AIAU 27607 (2007)

[23] D. Jung et al., Rev. Sci. Instrum. 82, 043301-1-5 (2011)

[24] G.P. Grim et al., Rev Sci. Instr. 75, 3572 (2004)

[25] D.B. Pelowitz textitet al., MCNPX User's Manual, Version 2.7.B LA-UR-09-04 150 (2009); http://menpx.lanl.gov/ 